\title{
Evaluation of clinical impact of pharmacogenomics knowledge involved in CPIC guidelines on Chinese pediatric patients
}

\author{
Weifeng Qin ${ }^{1,2}$, Zhenglin Du³, Jingfa Xiao ${ }^{3}$, Huilong Duan², Qiang Shu \& Haomin Li*,1 \\ ${ }^{1}$ The Children's Hospital, Zhejiang University School of Medicine and National Clinical Research Center for Child Health, Hangzhou \\ 310052, PR China \\ ${ }^{2}$ College of Biomedical Engineering and Instrument Science, Zhejiang University, Hangzhou 310027, PR China \\ ${ }^{3}$ Beijing Institute of Genomics, Chinese Academy of Sciences, Beijing 100101, PR China \\ *Author for correspondence: Tel.: +86 1386744 5504; Fax: +86 5718795 1960; hmli@zju.edu.cn
}

\begin{abstract}
Aim: To evaluate the clinical benefits of implementing pharmacogenomics testing for Chinese pediatric patients. Materials \& methods : Based on the drug-gene interactions involved in the Clinical Pharmacogenetics Implementation Consortium guidelines, whole-genome sequencing data from the Chinese Academy of Sciences Precision Medicine Initiative project and the medication data of pediatric patients from a children's hospital, the prevalence of the Chinese population with actionable pharmacogenomic variants was calculated, the prescribing pattern for pediatric patients was analyzed. Results: $37.0 \%$ of the drugs involved in the Clinical Pharmacogenetics Implementation Consortium guidelines were used by Chinese pediatric patients, $8.91 \%$ inpatients and $0.89 \%$ outpatients received at least one pharmacogenomics medication, $1.24 \%$ (4803) inpatients and $0.16 \%$ (2940) outpatients were estimated to be at high risk of pharmacogenomic-related adverse therapeutic outcomes. Conclusion: Implementing pharmacogenomics testing can improve therapeutic outcomes for many Chinese pediatric patients.
\end{abstract}

First draft submitted: 23 October 2019; Accepted for publication: 27 November 2019; Published online: 22 January 2020

Keywords: adverse drug reactions $\bullet$ EHR $\bullet$ pediatric $\bullet$ pharmacogenomics clinical implementation

Pharmacogenomics is responsible for much of the interindividual variability in drug responses, such as therapeutic failure and toxicity [1]. Clinical implementation of pharmacogenomics has the potential to optimize drug therapy, improve clinical outcomes and reduce clinical care costs [2], and it is the foremost way of realizing personalized or precision medicine $[3,4]$. Hundreds of drugs approved by the US FDA contain pharmacogenomic biomarkers in the drug labeling [3,5]. Germline pharmacogenomic variants affect a patient's care throughout life. Recent largescale genotyping and sequencing studies have found that more than $90 \%$ of subjects carry one or more clinically actionable pharmacogenomic variants that would influence drug therapy outcomes [6-8]. However, the translation of pharmacogenomic knowledge into clinical practice has been slow due to several long-standing unresolved barriers and challenges $[4,9,10]$.

Two collaborative initiatives, the NIH-funded Pharmacogenomics Knowledge Base (PharmGKB) and the Clinical Pharmacogenetics Implementation Consortium (CPIC), are leading efforts to curate and disseminate pharmacogenomics knowledge to accelerate the clinical translation [11,12]. Moreover, for the clinically actionable pharmacogenomics knowledge (gene-drug interactions assigned as Level A or Level B by CPIC), which supports at least one moderate or strong prescribing recommendation, the CPIC is making ongoing efforts to publish peer-reviewed, evidenced-based guidelines. To date, more than 100 gene-drug pairs have been identified as clinically actionable, and dozens of guidelines have been published for more than 40 drugs, including many commonly prescribed drugs, such as warfarin [13], voriconazole [14] and simvastatin [15]. However, the CPIC guidelines provide detailed genotype-based prescribing recommendations, they provide no guidance regarding when and how to execute pharmacogenomic tests [16]. It is also uncertain which pharmacogenomic tests have the greatest clinical utility for a specific population, especially for pediatric patients. A thorough assessment of the prevalence of pharmacogenomic variants may help identify tests with greater utility and facilitate the clinical translation of pharmacogenomics.

Future Medicine 
Table 1. Drugs and genes involved in the Clinical Pharmacogenetics Implementation Consortium guidelines.

\begin{tabular}{|l|l|}
\hline Drugs & Genes \\
\hline $\begin{array}{l}\text { Atomoxetine, codeine, desipramine, fluvoxamine, nortriptyline, ondansetron, } \\
\text { paroxetine, tamoxifen, tropisetron }\end{array}$ & CYP2D6 \\
\hline Citalopram, clopidogrel, escitalopram, sertraline, voriconazole & CYP2C19 \\
\hline Amitriptyline, clomipramine, doxepin, imipramine, trimipramine & CYP2D6, CYP2C19 \\
\hline $\begin{array}{l}\text { Desflurane, enflurane, halothane, isoflurane, methoxyflurane, sevoflurane, } \\
\text { succinylcholine }\end{array}$ & CACNA1S, RYR1 \\
\hline Peginterferon alfa-2a, peginterferon alfa-2b, ribavirin & IFNL3 \\
\hline Azathioprine, mercaptopurine, thioguanine & TPMT, NUDT15 \\
\hline Abacavir, allopurinol, oxcarbazepine & HLA-B \\
\hline Capecitabine, fluorouracil & DPYD \\
\hline Warfarin & CYP2C9, VKORC1, CYP4F2 \\
\hline Carbamazepine & HLA-A, HLA-B \\
\hline Phenytoin & CYP2C9, HLA-B \\
\hline Atazanavir & UGT1A1 \\
\hline Simvastatin & SLCO1B1 \\
\hline Tacrolimus & CYP3A5 \\
\hline Efavirenz & CYP2B6 \\
\hline Ivacaftor & CFTR \\
\hline Rasburicase & G6PD \\
\hline
\end{tabular}

Due to the lifelong useful nature of pharmacogenomic data, one may obtain more benefits from pharmacogenomic tests if such tests could be carried out as early as possible, for example, during childhood or at birth [5]. On the other hand, pediatric patients are always seen as a unique population in clinical drug use, for whom evidence-based dosing guidance is often absent, drug efficacy is unproven and toxicity is concerning. The incidence of adverse drug reactions (ADRs) in pediatric patients is more common relative to adult patients [17-20]. Because the organs and tissues of children are developing, the functions of their bodies are dynamic, leading to less experience and therapeutic data on pediatric medication for most drugs that can be referenced by clinicians, and ADRs are more likely to occur in children than in adults. Therefore, pharmacogenomic clinical testing, as an approach could help guide the choice of medication within and between classes of drugs where therapeutic alternatives exist and the selection of an initial dose, is especially important in pediatric practice. However, implementation of pharmacogenomic testing in pediatric care is still scarce [21], this may partly because many pharmacogenomics knowledge come from researches of adults and cannot extrapolated directly to children patients [21,22], and also because it is unclear to what extent drug inefficacy and adverse events could be mitigated by implementing pharmacogenomic testing in pediatric patients.

The purpose of this study is to evaluate the clinical impact of adopting pharmacogenomic tests for drugs involved in the CPIC guidelines within the Chinese pediatric population and to facilitate the clinical implementation of pharmacogenomics in China. Thus, we estimated the prevalence of clinically actionable pharmacogenomic variants among the Chinese population, identified the prescribed medications with CPIC guidelines among the pediatric population from the Electronic Health Record (EHR) system of a children's hospital, assessed the potential impact of actionable pharmacogenomic knowledge on the present prescribing pattern for the targeted population and discussed the pharmacogenomic test solutions that could be clinically adopted now and in the future.

\section{Materials \& methods}

This study was approved by The Children's Hospital Zhejiang University School of Medicine (CHZUSM), which is a Chinese tertiary level A children's hospital, and supported by the Beijing Institute of Genomics Chinese Academy of Sciences. The gene-drug interaction knowledge involved in the CPIC guidelines all have a relatively high level of clinical evidence, and each guideline provides genotype-based prescribing recommendations that are clinically actionable and can be handily used by clinicians to tailor therapy for patients in clinical settings. Therefore, all 65 clinically actionable gene-drug pairs involved in the CPIC guidelines, referring to 19 unique genes and 46 unique drugs (Table 1), were investigated in this study. 


\section{Prevalence estimation of actionable pharmacogenomics variants in Chinese population}

The allele frequencies of pharmacogenomic variants were collected from the Chinese Academy of Sciences Precision Medicine Initiative (CASPMI) variant call dataset (Supplementary Table 1). The CASPMI includes 991 healthy Chinese people's genomic data sequenced by whole-genome sequencing (WGS) with a mean depth of 30X (25X$35 \mathrm{X})$ [23]. The allele designation and definition, for example, the CYP450 star-allele nomenclature [24], is directly collected from the allele definition table curated by the PharmGKB website. For the HLA-A and $H L A-B$ star alleles and the CYP2D6 duplication alleles, it is too complex to exactly calculate the star allele frequency directly from the CASPMI variant call dataset, so we use the east Asian population frequency provided by the CPIC guidelines as an approximation and alternative to the Chinese population frequency. Alleles of the same gene were treated as mutually exclusive, and the frequency of the wild-type or default allele of normal function, for example, $C Y P 2 C 19^{*} 1$, was calculated as 1 minus the sum of the actionable allele frequencies. An actionable (risk) pharmacogenomics phenotype is defined as any phenotype that could cause the prescription of at least one drug to change, including drug alteration or dose adjustment, according to the CPIC dosing guidelines. All of the allele functions (Supplementary Table 1), actionable (risk) pharmacogenomic phenotypes and phenotype definitions (Supplementary Table 2) were manually collected from the CPIC guidelines. The prevalence of the actionable phenotype was calculated based on the Hardy-Weinberg equation, and an example of the calculation is shown in Supplementary Table 2.

Among all the actionable gene-drug pairs, there are many instances in which several drugs are affected by the same gene, while the actionable phenotypes of these drugs are not the same. For example, the CYP2C19 intermediate phenotype is actionable for clopidogrel but is not actionable for voriconazole, clomipramine and doxepin. Moreover, there are also many drugs affected by more than one gene. Therefore, the total prevalence of each pharmacogenomic drug was also estimated. In the calculation, phenotypes in different genes were treated as mutually independent, so the total prevalence of pharmacogenomic drugs affected by more than one gene was calculated as 1 minus the product of the probabilities of nonactionable phenotypes of all genes related to that drug; similarly, the proportion of the population who would carry at least one actionable phenotype was estimated as 1 minus the product of the probabilities of nonactionable phenotypes of all genes related.

\section{Statistics of prescription drugs for the pediatric population}

Until the start of this study, genotype-guided medication prescribing was not performed within the CHZUSM, and all patients' medication prescription statistics were not affected by their pharmacogenomic phenotype status. The medication data of pediatric patients were extracted from the EHR system of CHZUSM, which consists of two subsystems, in other words, the inpatient and outpatient subsystems. All of the inpatient medication data from July 2001 to June 2019 and all of the outpatient medication data from October 2010 to June 2019 were extracted from the two subsystems. Unique users were identified by the patient index ID, which is used in the EHR system of CHZUSM to uniquely identify each patient. Patients who ranged in age from 0 to 18 years and had at least one record for a medication in the EHR system were included in the analysis. We queried the unique pediatric patient users prescribed pharmacogenomic drugs involved in the CPIC guidelines in the inpatient and outpatient EHR subsystems. We estimated the proportion of patients exposed to pharmacogenomic medications and at risk of drug inefficacy or toxicity through the product of the total prevalence of pharmacogenomic drug and the number of patients exposed to that drug.

\section{Results}

The estimated prevalences of the actionable phenotypes in the Chinese population for all the genes involved in CPIC guidelines are shown in Supplementary Table 2. The CFTR, G6PD and CACNA1S genes are not listed in the table because no actionable variants were found in the sequenced CASPMI population samples. The most prevalent variant was in the VKORC1 gene, which influences the anticoagulant medication warfarin [13], with an estimated prevalence of actionable genotypes of up to $98.6 \%$. The estimated total prevalences of actionable phenotypes for all drugs involved in CPIC guidelines in the Chinese population are shown in Supplementary Table 3. It can be seen that for more than half of the drugs, the total prevalences of actionable phenotypes are greater than $10 \%$. This indicates that a considerable proportion of pharmacogenomics-related drug inefficacy and ADRs caused by these medications could be avoided or greatly reduced by the clinical implementation of pharmacogenomics tests.

Individuals with an actionable pharmacogenomic phenotype are considered at risk only if they are exposed to the associated medications. A total of 388,278 pediatric inpatients and 1,790,597 pediatric outpatients who had 
Table 2. Drugs involved in the Clinical Pharmacogenetics Implementation Consortium guidelines prescribed to pediatric patients, and their therapeutic areas, main concerns, number and proportion of drug users in inpatients and outpatients.

\begin{tabular}{|c|c|c|c|c|}
\hline Drug & Therapeutic area & Main concern & Inpatient drug users (\%) & Outpatient drug users (\%) \\
\hline Ondansetron & Oncology & Inefficacy & $27,650(7.12)$ & $1540(0.09)$ \\
\hline Mercaptopurine & Oncology & Toxicity & $7391(1.90)$ & $441(0.02)$ \\
\hline Voriconazole & Infectious disease & Inefficacy, toxicity & $6102(1.57)$ & $238(0.01)$ \\
\hline Oxcarbazepine & Neurology & Toxicity & $1259(0.32)$ & $3310(0.18)$ \\
\hline Thioguanine & Oncology & Toxicity & $1221(0.31)$ & $0(0.00)$ \\
\hline Tacrolimus & Immunology & Inefficacy & $1039(0.27)$ & $2357(0.13)$ \\
\hline Allopurinol & Endocrinology & Toxicity & $752(0.19)$ & $0(0.00)$ \\
\hline Carbamazepine & Neurology & Toxicity & $571(0.15)$ & $466(0.03)$ \\
\hline Clopidogrel & Cardiology & Inefficacy & $475(0.12)$ & $471(0.03)$ \\
\hline Azathioprine & Immunology & Toxicity & $232(0.06)$ & $140(0.01)$ \\
\hline Sertraline & Neurology & Inefficacy, toxicity & $222(0.06)$ & $2022(0.11)$ \\
\hline Fluorouracil & Oncology & Toxicity & $161(0.04)$ & $0(0.00)$ \\
\hline Warfarin & Cardiology & Toxicity & $153(0.04)$ & $59(0.00)$ \\
\hline Phenytoin & Neurology & Toxicity & $59(0.02)$ & $0(0.00)$ \\
\hline Simvastatin & Cardiology & Toxicity & $10(0.00)$ & $26(0.00)$ \\
\hline Succinylcholine & Anesthesiology & Toxicity & $10(0.00)$ & $0(0.00)$ \\
\hline Atomoxetine & Neurology & Toxicity & $3(0.00)$ & $4488(0.25)$ \\
\hline
\end{tabular}

at least one medication record were identified from the inpatient and outpatient EHR subsystems of CHZUSM, respectively. The mean age (standard deviation) for inpatients and outpatients were 4.21 years (4.01) and 3.66 years (3.34), respectively. A total of $61.2 \%$ of the inpatients and $56.2 \%$ of the outpatients were male. All patients belonged to the Chinese population.

Table 2 shows the pharmacogenomic drugs involved in the CPIC guidelines that were ever prescribed to pediatric patients. It could be seen that 17 drugs had ever been used by pediatric patients. By checking the drug labels (approved by the US FDA) of the 17 drugs, we found that for the drugs clopidogrel, azathioprine, fluorouracil and warfarin, the safety and effectiveness have not been established for pediatric use, for the other 13 drugs, the safety and effectiveness data mostly only come from limited controlled clinical trials and can only include partial ages of the pediatric population, this indicate that the off-label drug use is common in pediatric population for the drugs affected by pharmacogenomics involved in CPIC guidelines, and it is essential for clinicians to follow some guidelines to guide the use of these drugs in pediatric patients, including the CPIC guidelines if patients' genetic data are available. Inpatients used all 17 drugs, while outpatients used only 12 . For the inpatients, the most commonly used drug was ondansetron, and 27,650 inpatients had been prescribed this drug, accounting for 7.12\% of all inpatients; the least commonly used drug was atomoxetine, and only three inpatients had been prescribed it. For the outpatients, the most commonly used drug was atomoxetine, and 4488 outpatients had been prescribed it, accounting for $0.25 \%$ of all outpatients. The least commonly used drug was simvastatin, and it had been prescribed to 26 outpatients. Although only approximately $37.0 \%$ (17/46) of all the drugs involved in the CPIC guidelines had been used by children, these drugs were associated with $73.7 \%(14 / 19)$ of all the genes involved in the CPIC guidelines. Moreover, these 14 genes affected 84.8\% (39/46) of all the drugs involved in the CPIC guidelines.

Figure 1 shows the number and proportion of unique patients who used pharmacogenomics drugs involved in the CPIC guidelines. For inpatients (Figure 1A), 34,589 (8.91\%) patients had received at least one drug, 10,856 $(2.8 \%)$ patients had received at least two drugs and $2181(0.56 \%)$ patients had received at least three drugs. For outpatients (Figure 1B), 15,862 (0.89\%) patients had received at least one drug, $598(0.03 \%)$ patients had received at least two drugs and only $37(0.002 \%)$ patients had received at least three drugs.

The number of patients used drugs involved in CPIC guidelines among therapeutic areas was calculated and is shown in Figure 2. The oncology drugs and the neurology drugs have the largest numbers of drug users among the inpatients and outpatients, respectively. The users of these drugs were 36,423 and 10,286, respectively. The cardiology drugs had a lower proportion of drug users among both the inpatients and outpatients, and they only had 638 and 556 drug users, respectively. 


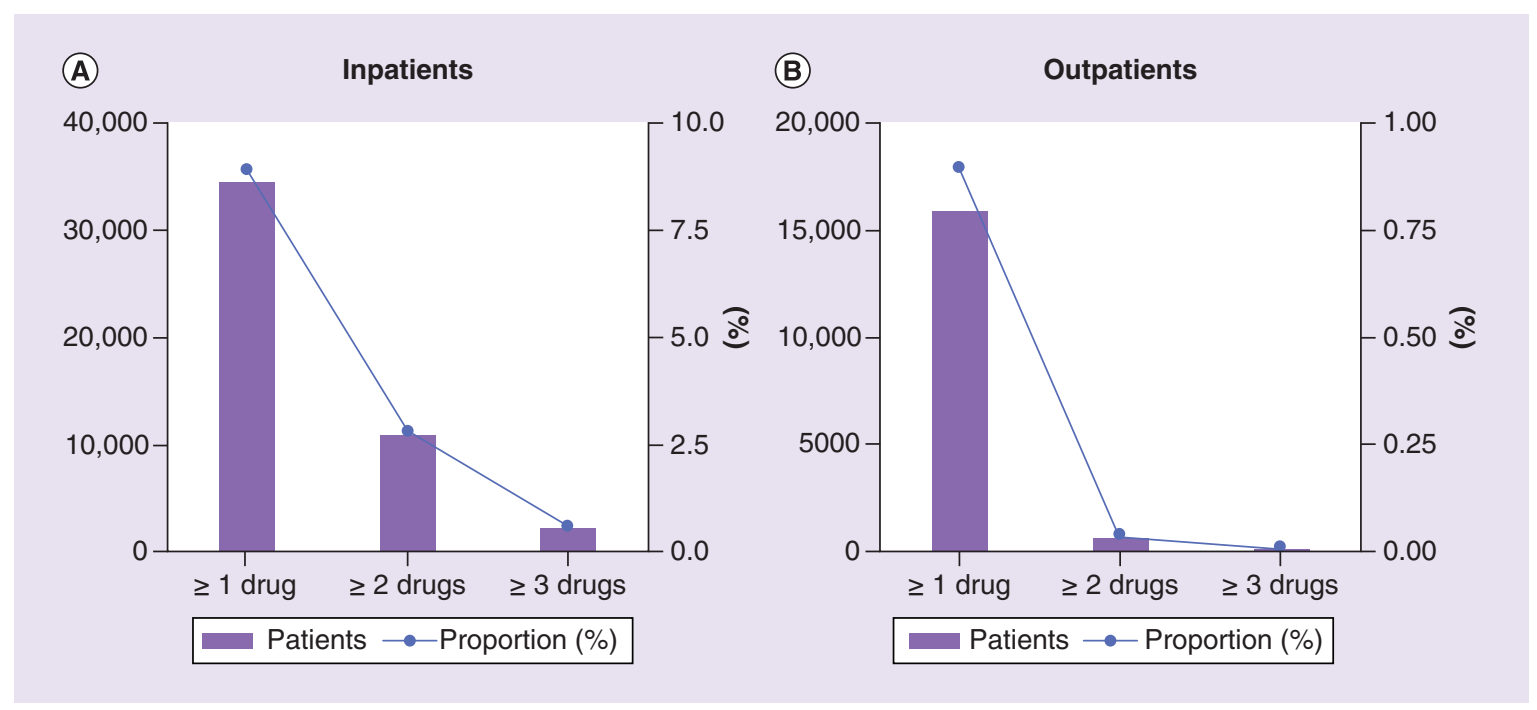

Figure 1. Number and proportion of patients used one or more drugs involved in the Clinical Pharmacogenetics Implementation Consortium guidelines. (A) For inpatients; (B) For outpatients.

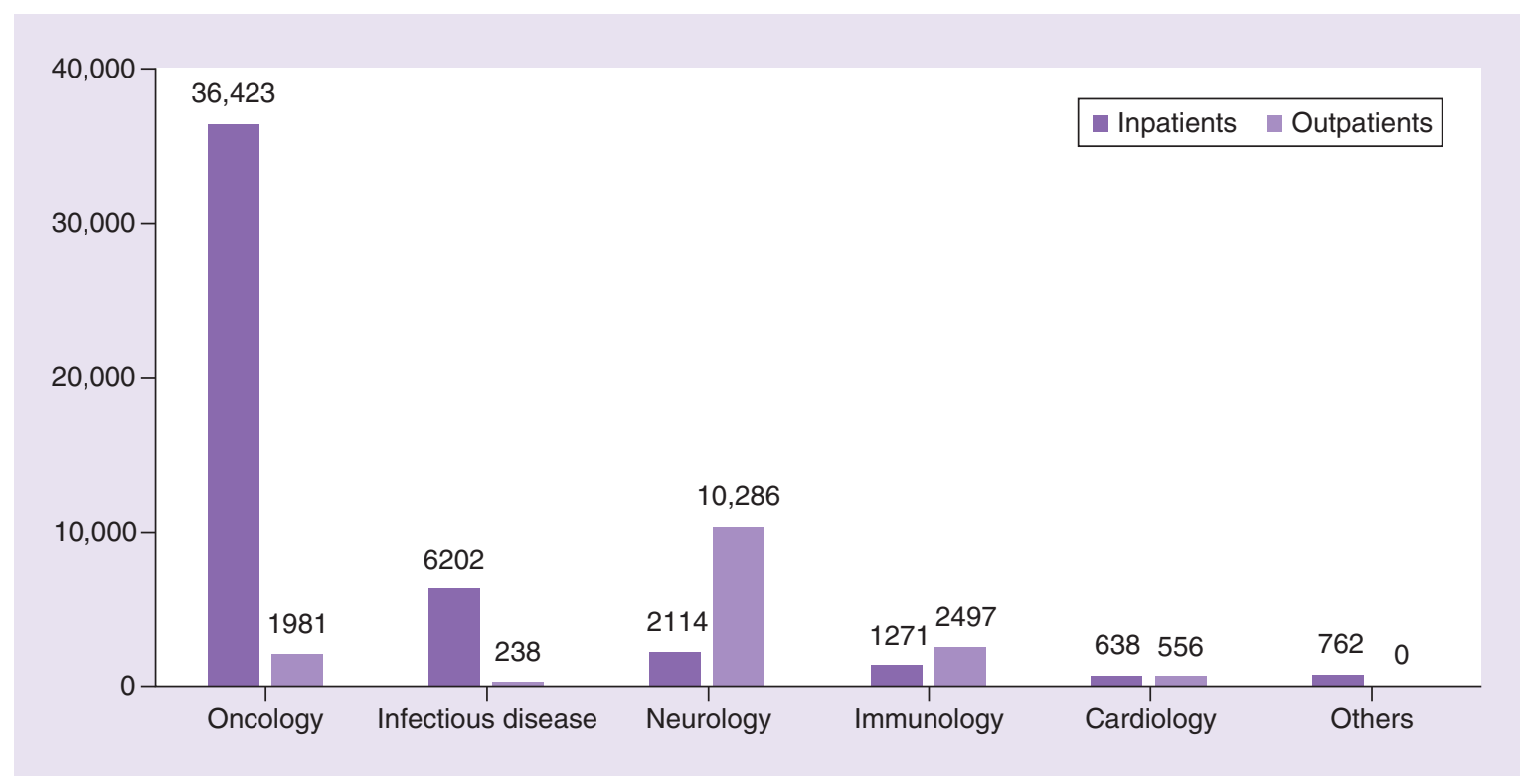

Figure 2. Number of patients used drugs involved in the Clinical Pharmacogenetics Implementation Consortium guidelines among therapeutic areas.

We estimated the numbers of patients potentially at risk of adverse therapeutic outcomes, including therapeutic failure and ADRs, and the need to adjust dosing or alter the drug by determining the total prevalence of actionable genotypes for each drug in exposed patients. As shown in Table 3, among 7391 inpatient children receiving mercaptopurine, the total prevalence of those affected by the TPMT and NUDT15 genes was 25.27\%, and 1868 $(0.48 \%$ of all pediatric inpatients) patients were estimated to have an increased risk of pharmacogenomic-related leukopenia, neutropenia, myelosuppression at the standard dosing and needed to reduce the dosing or alter the drug. Among 2357 outpatient children-receiving tacrolimus, the total prevalence of those affected by the CYP3A5 gene was $48.59 \%$, and 1145 (0.06\% of all pediatric outpatients) patients were estimated to have a decreased chance of achieving target concentrations at the standard dosing and needed to increase the dose. The total prevalence of all the drugs involved in the CPIC guidelines with the related genes and the calculation method of the total prevalence are shown in Supplementary Table 3. In addition, the number of clopidogrel recipients includes all pediatric 
Table 3. Estimated number of inpatients and outpatients with risk of drug inefficacy or toxicity, and prevalence of actionable phenotypes for drugs involved in the Clinical Pharmacogenetics Implementation Consortium guidelines and prescribed to pediatric patients.

\begin{tabular}{|c|c|c|c|c|}
\hline Drug & Genes & Prevalence (\%) & Inpatient with risk (\%) & Outpatients with risk (\%) \\
\hline Mercaptopurine & TPMT, NUDT15 & 25.27 & $1868(0.48)$ & $111(0.01)$ \\
\hline Voriconazole & CYP2C19 & 14.13 & $862(0.22)$ & $34(0.00)$ \\
\hline Tacrolimus & CYP3A5 & 48.59 & $505(0.13)$ & $1145(0.06)$ \\
\hline Ondansetron & CYP2D6 & 1.30 & $359(0.09)$ & $20(0.00)$ \\
\hline Thioguanine & TPMT, NUDT15 & 25.27 & $309(0.08)$ & $0(0.00)$ \\
\hline Clopidogrel & CYP2C19 & 58.95 & $280(0.07)$ & $278(0.02)$ \\
\hline Oxcarbazepine & $H L A-B$ & 13.28 & $167(0.04)$ & $440(0.02)$ \\
\hline Warfarin & CYP2C9, VKORC1, CYP4F2 & 99.58 & $152(0.04)$ & $59(0.00)$ \\
\hline Carbamazepine & $H L A-A, H L A-B$ & 18.97 & $108(0.03)$ & $88(0.00)$ \\
\hline Allopurinol & $H L A-B$ & 11.89 & $89(0.02)$ & $0(0.00)$ \\
\hline Azathioprine & TPMT, NUDT15 & 25.27 & $59(0.02)$ & $35(0.00)$ \\
\hline Sertraline & CYP2C19 & 14.13 & $31(0.01)$ & $286(0.02)$ \\
\hline Phenytoin & $H L A-B, C Y P 2 C 9$ & 21.15 & $12(0.00)$ & $0(0.00)$ \\
\hline Simvastatin & SLCO1B1 & 22.03 & $2(0.00)$ & $6(0.00)$ \\
\hline Fluorouracil & $D P Y D$ & 0.20 & $0(0.00)$ & $0(0.00)$ \\
\hline Succinylcholine & CACNA1S, RYR1 & 0.20 & $0(0.00)$ & $0(0.00)$ \\
\hline Atomoxetine & CYP2D6 & 9.75 & $0(0.00)$ & $438(0.02)$ \\
\hline Total & & & 4803 (1.24) & $2940(0.16)$ \\
\hline
\end{tabular}

patients-receiving clopidogrel, not only those receiving it after Percutaneous Coronary Intervention. Given that the CPIC guidelines suggest that the recommendations are only applicable for patients after Percutaneous Coronary Intervention, the number of children taking clopidogrel with antiplatelet failure should be fewer than the estimated number.

By tabulating the frequencies of actionable phenotypes, $99.98 \%$ of the Chinese population were estimated to have at least one actionable pharmacogenomic phenotype, as shown in Supplementary Table 4; excluding the genes that do not affect drugs used in Chinese children, $99.97 \%$ of Chinese children were estimated to have at least one actionable pharmacogenomic phenotype, as shown in Supplementary Table 5. Even excluding the genes affecting warfarin, for which almost all Chinese people have actionable phenotypes (99.6\%) and can benefit from related pharmacogenomic information, the above two prevalence rates are still as high as to 96.26 and $93.26 \%$, respectively. Our estimates based on population-specific allele frequencies are consistent with previous studies directly genotyping or sequencing large biobank populations $[6,7]$, demonstrating that pharmacogenomic variants are found in more than $90 \%$ of the population.

\section{Discussion}

To the best of our knowledge, this is the first study to evaluate the clinical benefits that the Chinese pediatric population could obtain from the clinical translation of pharmacogenomic knowledge involved in the CPIC guidelines. Almost all Chinese carry at least one actionable pharmacogenomic genotype that could influence pharmacotherapy decisions when they are prescribed a drug involved in the CPIC guidelines. Approximately $8.91 \%$ of inpatients and $0.89 \%$ of outpatients received at least one drug involved in the CPIC guidelines. Although only $37.0 \%$ (17/46) of the CPIC drugs are being used in pediatric patients, these drugs are associated with $73.7 \%$ (14/19) of all CPIC guideline-involved genes. Moreover, these 14 genes affect $84.8 \%(39 / 46)$ of all the drugs involved in the CPIC guidelines. A total of $4803(1.24 \%)$ inpatients and $2940(0.16 \%)$ outpatients were estimated to be at high risk for pharmacogenomic-related adverse therapeutic outcomes. For more than half of the drugs involved in the CPIC guidelines, the prevalence of actionable phenotypes was more than $10 \%$. These results suggest that initial testing for a panel of relevant pharmacogenomic variants among children or newborns in the Chinese population may inform pharmacotherapy decisions over their entire lifetime of care, and the adverse therapeutic outcomes in Chinese pediatric patients could be considerably reduced by implementing pharmacogenomics tests. 
This study provides information that can help healthcare and clinical leadership in China develop a strategy and framework for the comprehensive clinical implementation of CPIC guidelines.

In 2016, Samwald et al. [25] studied the prescribing pattern of pharmacogenomic drugs in American pediatric patients between 0 and 14 years old by examining the healthcare administrative medical claims data from American commercial insurers, and they found that $11.2 \%$ of pediatric patients had received at least one pharmacogenomic drug. This drug exposure result was higher than ours ( $8.91 \%$ in inpatients and $0.89 \%$ in outpatients), which may be due to two reasons. One is that they investigated more drugs, not just those involved in the CPIC guidelines but also including those involved in the Dutch Pharmacogenomics Working Group guidelines, while we only investigated drugs involved in the CPIC guidelines. The other is the difference in drug regulatory policies between the two countries; for example, codeine is the most commonly used drug prescribed to children according to Samwald et al.'s study, while the Chinese government decided to forbid doctors to prescribe codeine-containing drugs to patients under 18 years old in 2018. Moreover, our drug exposure result in the pediatric population is much lower than the results found in the adult population $(>30 \%)$ [25-27]. In addition to their medication lists being larger among adults, the more important reason is the prevalence of some diseases, such as cardiovascular disease, is much higher in older adults than in children. This leads to the extremely high exposure to cardiovascular drugs in adults, as reported by Chanfreau-Coffinier $e$ t al., while cardiovascular drugs only have a very limited number of users among pediatric patients, as shown in Figure 2. Although drug exposure is lower in children than in adults, given the clinical impact of the adverse therapeutic outcomes on pediatric patients and the lifetime usefulness of pharmacogenomic test results, testing for a panel of pharmacogenomic variants in pediatric populations may obtain more benefits compared with testing later in life.

Two main models have been used to implement pharmacogenomic tests for patients in clinical practice. One is the currently widely used reactive pharmacogenomic testing model, in other words, when a clinician considers ordering a drug affected by actionable pharmacogenomics, the relative pharmacogenomic test is reactively ordered for the patient. Although this model allows clinicians to select pharmacogenomic tests with higher levels of evidence and avoid unnecessary testing, it also has several limitations. The tests must be ordered by the clinicians at the point of care in the clinical setting, and the test results must be returned in a timely manner to avoid delaying the prescription for patients too long [5,27].

The other model is the preemptive pharmacogenomic testing model, and several centers have launched pilot programs to implement this testing model in clinical practice [28-32]. In this model, a group of people are first enrolled with consent; then, a panel of pharmacogenomic variants are genotyped preemptively for these people, and the genotyping results are stored in the EHR for future use. When some day one individual is prescribed drugs affected by the pharmacogenomic results, these results and the prescribing recommendations are provided together to the clinician with the help of the Clinical Decision Support (CDS) system to guide the clinician in tailoring the prescription. The preemptive pharmacogenomic testing model is a suitable solution to be implemented in Chinese pediatric patients for personalized medicine based on the above analysis results. This model solves the limitations that exist in the reactive model, but new challenges arise with this model. First, it is difficult to determine which gene-drug interactions should be included in the pharmacogenomic testing, especially for those without clinical practice guidelines [30,33]. Second, it is necessary to identify which individuals would benefit from the preemptive testing and should therefore be enrolled in the program [30]. For example, Vanderbilt University used a prognostic model to overcome this challenge [34]. Third, it is difficult to integrate the pharmacogenomic results into the EHR because current commercial EHR systems are not designed to store genomic information in a format suitable for long-term use, and the pharmacogenomic results are usually provided as unstructured text in a PDF file by the genomic test laboratory [33]. They usually need the help of other auxiliary tools to translate or exchange in different systems, many efforts have been made to overcome this challenge, and some of these efforts have formed practical solutions that could be used by other institutions $[35,36]$. Fourth, with more pharmacogenomics information emerging in the clinical setting under preemptive testing model, current providers lack pharmacogenomics knowledge [37], this may causing the potential of misinterpretation of the testing results and even leading to medical safety problems, so it needs elaborately designed education and CDS solutions to avoid such things at the initiating of the preemptive testing [33,38]. Moreover, when more pharmacogenomic evidence and new guidelines become available and prescribing patterns change, it will be challenging to maintain the knowledge bases of pharmacogenomics CDS tools to keep them current [38,39].

Given to the above challenges accompanied with preemptive model, the reactive model would not be completely replaced in a short term by the preemptive model. And moreover, based on the result showed in Figure 2, the 
mostly used drug substances by pediatric inpatients are for the therapy of oncological diseases that have a high mortality rate relative to other diseases, for patients with such high mortality diseases the reactive testing model may be more suitable and reasonable than the preemptive testing model which tests a panel of genes once a time. In recent years, many gene testing companies have begun to provide commercial pharmacogenomic testing for clinical use in China, including many clinical significant pharmacogenes with CPIC guidelines, such as TPMT, NUDT15, CYP2C19 - among others. However, due to these commercial testing services usually being very expensive and needing a long turnaround time (i.e., about 10 days to return the testing results), they are not likely to be adopted by the hospital as routine testing. As of the beginning of this study, there was not any kind of pharmacogenomics testing routinely performed in the CHZUSM. According to these facts and the result of this study, it might be reasonable for many of China's hospital to routinely implement the reactive gene testing for some specific diseases and drugs, such as testing TPMT and NUDT15 for pediatric patients with acute lymphoblastic leukemia need to use mercaptopurine, before the preemptive testing model is widely implemented in China. But, from the long-term perspective, with the challenges and barriers of preemptive model gradually being overcome, the preemptive testing model is undoubtedly still the best choice for most of hospitals in China, even if the reactive testing model would exist in parallel in a long period.

In addition to the above two models that are currently in clinical use, next-generation sequencing (NGS), for example, WGS and whole exome sequencing, are being adopted in clinical use. The use of WGS and whole-exome sequencing for diagnosis in children with neurodevelopmental disabilities is rapidly becoming the standard of care in light of recent reports [40,41]. Making use of incidental pharmacogenomic findings from exome sequencing to develop CDS alerts has also been reported [42]. NGS, especially WGS, is a promising way of replacing the reactive and preemptive testing models and supports the wide implementation of precision medicine because it is not only sufficiently accurate but also faster and less expensive per base pair compared with the current widely used genotyping test. However, many unresolved barriers block the wide clinical implementation of WGS. Although the detailed discussion of these barriers is beyond the scope of this study, one of the most important obstacles to the wide use of WGS is that the data are too large: one person's genome contains approximately 3 billion base pairs. Thus, this large amount of data even for one person exceeds the storage, retrieval and processing capacity of any EHR and CDS system currently in use. Despite the many challenges and barriers in each model, we still believe that the wide clinical implementation of pharmacogenomics and personalized precision medicine will soon occur.

\section{Limitations}

Several limitations of this study should be noted. This study is not a well-controlled clinical study and we use the real-world prescription data of pediatric inpatients and outpatients with various diseases, the estimated proportion of patients who can benefit from pharmacogenomics testing may vary greatly from disease to disease and region to region. Given the relatively small size of the CASPMI samples, we may not have included some rare pharmacogenomic variants. We did not examine outcomes associated with prescribing drugs involved in the CPIC guidelines in pediatric patients with high-risk phenotypes. Additionally, the landscape of medication exposure is rapidly changing, and we did not report the medication trends over time. There might be minor differences in prescribing patterns within different hospitals; this study only reflects the prescriptions of drugs involved in the CPIC guidelines used in a typical Chinese children's hospital, the CHZUSM. This study included drugs involved in the CPIC guidelines and did not include drugs with high-level evidence but without a CPIC recommendation or those involved in other pharmacogenomic guidelines, such as the Dutch Pharmacogenomics Working Group.

\section{Conclusion}

We estimated that clinically important pharmacogenomic variants are highly prevalent in the Chinese population, and almost all Chinese carry at least one actionable pharmacogenomic variant. In prescriptions for children, approximately $8.91 \%$ of inpatients and $0.89 \%$ of outpatients used the CPIC actionable drugs, accounting for a third of all the CPIC actionable drugs, and associating with $73.7 \%$ of all the genes involved in the CPIC guidelines. Many adverse therapeutic outcomes that occurred during the study period in inpatients and outpatients could have been avoided by implementing pharmacogenomic testing. These results suggest that initial testing for a panel of relevant pharmacogenomic variants, in other words, preemptive pharmacogenomic testing, among children or newborns in China may inform pharmacotherapy decisions over their entire lifetimes, improving therapeutic outcomes and reducing treatment costs for many Chinese pediatric patients, especially for inpatients. 


\section{Future perspective}

An initial testing for a panel of relevant pharmacogenomic variants, in other words, preemptive pharmacogenomic testing, among children or newborns in China can inform pharmacotherapy decisions over their entire lifetimes, improving therapeutic outcomes and reducing treatment costs for many Chinese pediatric patients. However, given the challenges discussed in the discussion part of this article, the preemptive pharmacogenomic testing model is not a scalable and sustainable model to be widely adopted by other developing areas and countries, such as China. NGS, especially WGS, is a promising way of replacing the reactive and preemptive testing models and supports the wide implementation of precision medicine. To date, NGS has come down to a very low price with enough accuracy for clinical use, but there is less actionable genomics knowledge available for clinical use, and the healthcare information technology infrastructure also do not support the clinical use of NGS data very well. Hence, research focusing on clinical translation of genomic knowledge, and aiming at surmounting challenges related to NGS data clinical storage, retrieval and NGS-based CDS, are the key to facilitate the clinical implementation of genomic knowledge in the near future.

\section{Summary points}

- Almost all Chinese carry at least one actionable pharmacogenomic genotype that could influence pharmacotherapy decisions when they are prescribed a drug involved in the CPIC guidelines.

- Approximately $8.91 \%$ of inpatients and $0.89 \%$ of outpatients received at least one drug involved in the CPIC guidelines. Although only $37.0 \%$ (17/46) of the CPIC drugs are being used in pediatric patients, these drugs are associated with $73.7 \%(14 / 19)$ of all CPIC guideline-involved genes. Moreover, these 14 genes affect $84.8 \%$ (39/46) of all the drugs involved in CPIC guidelines.

- A total of $4803(1.24 \%)$ inpatients and $2940(0.16 \%)$ outpatients were estimated to be at high risk for pharmacogenomic-related adverse therapeutic outcomes.

- For more than half of the drugs involved in the CPIC guidelines, the prevalence of actionable phenotypes was more than $10 \%$. These results suggest that initial testing for a panel of relevant pharmacogenomic variants among children or newborns in the Chinese population may inform pharmacotherapy decisions over their entire lifetime of care, and the adverse therapeutic outcomes in Chinese pediatric patients could be considerably reduced by implementing pharmacogenomics tests.

- This study provides information that can help healthcare and clinical leadership in China develop a strategy and framework for the comprehensive clinical implementation of CPIC guidelines.

\section{Author contributions}

W Qin, H Duan, Q Shu and H Li wrote the manuscript. H Li, W Qin, H Duan and Q Shu designed the research. H Li, W Qin and Q Shu collected the clinical data. Z Du, J Xiao and H Li collected the genomic data. W Qin, H Duan, Q Shu and H Li analyzed the data.

Financial \& competing interests disclosure

H Li was supported by the National Natural Science Foundation of China (81871456), National Key R\&D Program of China (2016YFC0901905; 2016YFC0901703), the Fundamental Research Funds for the Central Universities (2019XZZX003-16). The authors have no other relevant affiliations or financial involvement with any organization or entity with a financial interest in or financial conflict with the subject matter or materials discussed in the manuscript apart from those disclosed.

No writing assistance was utilized in the production of this manuscript.

\section{Open access}

This work is licensed under the Attribution-NonCommercial-NoDerivatives 4.0 Unported License. To view a copy of this license, visit http://creativecommons.org/licenses/by-nc-nd/4.0/

\section{References}

Papers of special note have been highlighted as: $\bullet$ of interest $\bullet \bullet$ of considerable interest

1. Evans WE, Relling MV. Pharmacogenomics: translating functional genomics into rational therapeutics. Science 286(5439), 487 (1999).

2. Verbelen M, Weale ME, Lewis CM. Cost-effectiveness of pharmacogenetic-guided treatment: are we there yet? Pharmacogenomics J. 17, 395 (2017).

- This review assessed published economic evaluations comparing the cost-effectiveness of pharmacogenomics-guided treatment to standard treatment, the result shows that pharmacogenomics has a positive impact on healthcare quality and costs. 
3. Dickmann LJ, Ware JA. Pharmacogenomics in the age of personalized medicine. Drug Discov. Today Technol. 21-22, 11-16 (2016).

4. Klein ME, Parvez MM, Shin J-G. Clinical implementation of pharmacogenomics for personalized precision medicine: barriers and solutions. J. Pharm. Sci. 106(9), 2368-2379 (2017).

5. Relling MV, Evans WE. Pharmacogenomics in the clinic. Nature 526(7573), 343-350 (2015).

-. This review elaborated the significance and evolution of pharmacogenomics in the clinic, delineated the main challenges and progress of current clinical implementation of pharmacogenomics.

6. Van Driest S, Shi Y, Bowton E et al. Clinically actionable genotypes among 10,000 patients with preemptive pharmacogenomic testing. Clin. Pharmacol. Ther. 95(4), 423-431 (2014).

7. Bush W, Crosslin D, Owusu-Obeng A et al. Genetic variation among 82 pharmacogenes: the PGRNseq data from the eMERGE network. Clin. Pharmacol. Ther. 100(2), 160-169 (2016).

- Variation within the key pharmacogenes in an eMERGE-PGx dataset was examined, and it was found that most individuals having multiple clinically actionable pharmacogenetic variants.

8. Ji Y, Skierka JM, Blommel JH et al. Preemptive pharmacogenomic testing for precision medicine: a comprehensive analysis of five actionable pharmacogenomic genes using next-generation DNA sequencing and a customized CYP2D6 genotyping cascade. J. Mol. Diagnos. 18(3), 438-445 (2016).

9. Lam YW. Scientific challenges and implementation barriers to translation of pharmacogenomics in clinical practice. ISRN Pharmacol. 2013(1), 641089 (2013).

10. Crews KR, Hicks JK, Pui C-H, Relling MV, Evans WE. Pharmacogenomics and individualized medicine: translating science into practice. Clin. Pharmacol. Ther. 92(4), 467-475 (2012).

11. Relling M, Klein T. CPIC: Clinical Pharmacogenetics Implementation Consortium of the Pharmacogenomics Research Network. Clin. Pharmacol. Ther. 89(3), 464-467 (2011).

12. Whirl-Carrillo M, Mcdonagh EM, Hebert JM et al. Pharmacogenomics knowledge for personalized medicine. Clin. Pharmacol. Ther. 92(4), 414-417 (2012).

13. Johnson J, Caudle K, Gong L et al. Clinical Pharmacogenetics Implementation Consortium (CPIC) guideline for pharmacogenetics-guided warfarin dosing: 2017 update. Clin. Pharmacol. Ther. 102(3), 397-404 (2017).

14. Moriyama B, Obeng AO, Barbarino J et al. Clinical Pharmacogenetics Implementation Consortium (CPIC) guidelines for CYP2C19 and voriconazole therapy. Clin. Pharmacol. Ther. 102(1), 45-51 (2017).

15. Ramsey LB, Johnson SG, Caudle KE et al. The Clinical Pharmacogenetics Implementation Consortium guideline for SLCO1B1 and simvastatin-induced myopathy: 2014 update. Clin. Pharmacol. Ther. 96(4), 423-428 (2014).

16. Caudle KE, Klein TE, Hoffman JM et al. Incorporation of pharmacogenomics into routine clinical practice: the Clinical Pharmacogenetics Implementation Consortium (CPIC) guideline development process. Curr. Drug Metab. 15(2), 209-217 (2014).

17. Blake KV, Zaccaria C, Domergue F, La Mache E, Saint-Raymond A, Hidalgo-Simon A. Comparison between paediatric and adult suspected adverse drug reactions reported to the European Medicines Agency: implications for pharmacovigilance. Pediatr. Drugs 16(4), 309-319 (2014).

18. Clavenna A, Bonati M. Adverse drug reactions in childhood: a review of prospective studies and safety alerts. Arch. Dis. Child. 94(9), 724 (2009).

19. Kunac DL, Kennedy J, Austin N, Reith D. Incidence, preventability, and impact of adverse drug events (ADEs) and potential ADEs in hospitalized children in New Zealand. Pediatr. Drugs 11(2), 153-160 (2009).

20. Eshetie TC, Hailemeskel B, Mekonnen N, Paulos G, Mekonnen AB, Girma T. Adverse drug events in hospitalized children at Ethiopian University Hospital: a prospective observational study. BMC Pediatr. 15(1), 83 (2015).

21. Maagdenberg H, Vijverberg SJH, Bierings MB et al. Pharmacogenomics in pediatric patients: towards personalized medicine. Pediatr. Drugs 18(4), 251-260 (2016).

22. Korbel L, George M, Kitzmiller J. Clinically relevant pharmacogenomic testing in pediatric practice. Clin. Pediatr. (Phila) 53(9), 831-838 (2014).

23. Du Z, Ma L, Qu H et al. Whole genome analyses of Chinese Population and De Novo assembly of a Northern Han Genome. Genomics, Proteomics Bioinformatics 17(3), 229-247 (2019).

24. Sim SC, Ingelman-Sundberg M. The human cytochrome P450 (CYP) allele nomenclature website: a peer-reviewed database of CYP variants and their associated effects. Hum. Genomics 4(4), 278-281 (2010).

25. Samwald $\mathrm{M}, \mathrm{Xu} \mathrm{H}$, Blagec $\mathrm{K}$ et al. Incidence of exposure of patients in the United States to multiple drugs for which pharmacogenomic guidelines are available. PLoS ONE 11(10), e0164972 (2016).

-. Using large claims datasets to examine the potential clinical impact of preemptive pharmacogenomics testing by examining the incidence of exposure to multiple pharmacogenopmics drugs in different age periods of patient populations.

26. Chanfreau-Coffinier C, Hull LE, Lynch JA et al. Projected prevalence of actionable pharmacogenetic variants and level A drugs prescribed among US veterans health administration pharmacy users. JAMA Netw. Open 2(6), e195345 (2019). 
- An estimate of the number of veterans who could benefit from implementation of pharmacogenetic testing in clinical care.

27. Schildcrout JS, Denny JC, Bowton E et al. Optimizing drug outcomes through pharmacogenetics: a case for preemptive genotyping. Clin. Pharmacol. Ther. 92(2), 235-242 (2012).

- Assessment of the potential benefits of prospective measurement of pharmacogenetic variants across multiple genes simultaneously to guide future drug therapy.

28. Bielinski SJ, Olson JE, Pathak J et al. Preemptive genotyping for personalized medicine: design of the right drug, right dose, right time - using genomic data to individualize treatment protocol. Mayo Clin. Proc. 89(1), 25-33 (2014).

29. Gottesman O, Scott SA, Ellis SB et al. The CLIPMERGE PGx Program: clinical implementation of personalized medicine through electronic health records and genomics-pharmacogenomics. Clin. Pharmacol. Ther. 94(2), 214-217 (2013).

30. Pulley JM, Denny JC, Peterson JF et al. Operational implementation of prospective genotyping for personalized medicine: the design of the Vanderbilt PREDICT project. Clin. Pharmacol. Ther. 92(1), 87-95 (2012).

- The design of the Vanderbilt PREDICT project, and the gleaned lessons from the operational implementation of the project.

31. O'donnell PH, Bush A, Spitz J et al. The 1200 patients project: creating a new medical model system for clinical implementation of pharmacogenomics. Clin. Pharmacol. Ther. 92(4), 446-449 (2012).

32. Hoffman JM, Haidar CE, Wilkinson MR et al. PG4KDS: a model for the clinical implementation of pre-emptive pharmacogenetics. Am. J. Med. Genet. C Semin. Med. Genet. 166C(1), 45-55 (2014).

33. Caraballo PJ, Hodge LS, Bielinski SJ et al. Multidisciplinary model to implement pharmacogenomics at the point of care. Genet. Med. 19, 421 (2017).

- A generalizable pharmacogenomics implementation model consisting of core components for initial use by the Mayo Clinic but also applicable and transferable to other institutions regardless of size or available infrastructure.

34. Schildcrout JS, Shi Y, Danciu I et al. A prognostic model based on readily available clinical data enriched a pre-emptive pharmacogenetic testing program. J. Clin. Epidemiol. 72, 107-115 (2016).

35. Van Der Wouden C, Cambon-Thomsen A, Cecchin E et al. Implementing pharmacogenomics in Europe: design and implementation strategy of the Ubiquitous Pharmacogenomics Consortium. Clin. Pharmacol. Ther. 101(3), 341-358 (2017).

36. Blagec K, Koopmann R, Crommentuijn-Van Rhenen M et al. Implementing pharmacogenomics decision support across seven European countries: the Ubiquitous Pharmacogenomics (U-PGx) project. J. Am. Med. Inform. Assoc. 25(7), 893-898 (2018).

37. Johansen Taber KA, Dickinson BD. Pharmacogenomic knowledge gaps and educational resource needs among physicians in selected specialties. Pharmacogenomics Pers. Med. 7, 145-162 (2014).

38. Caraballo PJ, Bielinski SJ, St Sauver JL, Weinshilboum RM. Electronic medical record-integrated pharmacogenomics and related clinical decision support concepts. Clin. Pharmacol. Ther. 102(2), 254-264 (2017).

39. Hicks JK, Dunnenberger HM, Gumpper KF, Haidar CE, Hoffman JM. Integrating pharmacogenomics into electronic health records with clinical decision support. Am. J. Health Syst. Pharm. 73(23), 1967-1976 (2016).

40. Soden SE, Saunders CJ, Willig LK et al. Effectiveness of exome and genome sequencing guided by acuity of illness for diagnosis of neurodevelopmental disorders. Sci. Transl. Med. 6(265), 265ra168 (2014).

41. Wright CF, Fitzgerald TW, Jones WD et al. Genetic diagnosis of developmental disorders in the DDD study: a scalable analysis of genome-wide research data. Lancet 385(9975), 1305-1314 (2015).

42. Nishimura AA, Shirts BH, Dorschner MO et al. Development of clinical decision support alerts for pharmacogenomic incidental findings from exome sequencing. Genet. Med. 17(11), 939-942 (2015). 
